\title{
Confidence Estimation for Information Extraction
}

\author{
Aron Culotta \\ Department of Computer Science \\ University of Massachusetts \\ Amherst, MA 01003 \\ culotta@cs.umass. edu
}

\author{
Andrew McCallum \\ Department of Computer Science \\ University of Massachusetts \\ Amherst, MA 01003 \\ mccallumecs . umass . edu
}

\begin{abstract}
Information extraction techniques automatically create structured databases from unstructured data sources, such as the Web or newswire documents. Despite the successes of these systems, accuracy will always be imperfect. For many reasons, it is highly desirable to accurately estimate the confidence the system has in the correctness of each extracted field. The information extraction system we evaluate is based on a linear-chain conditional random field (CRF), a probabilistic model which has performed well on information extraction tasks because of its ability to capture arbitrary, overlapping features of the input in a Markov model. We implement several techniques to estimate the confidence of both extracted fields and entire multi-field records, obtaining an average precision of $98 \%$ for retrieving correct fields and $87 \%$ for multi-field records.
\end{abstract}

\section{Introduction}

Information extraction usually consists of tagging a sequence of words (e.g. a Web document) with semantic labels (e.g. Personname, Phonenumber) and depositing these extracted fields into a database. Because automated information extraction will never be perfectly accurate, it is helpful to have an effective measure of the confidence that the proposed database entries are correct. There are at least three important applications of accurate confidence estimation. First, accuracy-coverage trade-offs are a common way to improve data integrity in databases. Efficiently making these trade-offs requires an accurate prediction of correctness.

Second, confidence estimates are essential for interactive information extraction, in which users may correct incorrectly extracted fields. These corrections are then automatically propagated in order to correct other mistakes in the same record. Directing the user to the least confident field allows the system to improve its performance with a minimal amount of user effort. Kristjannson et al. (2004) show that using accurate confidence estimation reduces error rates by $46 \%$.

Third, confidence estimates can improve performance of data mining algorithms that depend upon databases created by information extraction systems (McCallum and Jensen, 2003). Confidence estimates provide data mining applications with a richer set of "bottom-up" hypotheses, resulting in more accurate inferences. An example of this occurs in the task of citation co-reference resolution. An information extraction system labels each field of a paper citation (e.g. Author, TITLE), and then co-reference resolution merges disparate references to the same paper. Attaching a confidence value to each field allows the system to examine alternate labelings for less confident fields to improve performance.

Sound probabilistic extraction models are most conducive to accurate confidence estimation because of their intelligent handling of uncertainty information. In this work we use conditional random fields (Lafferty et al., 2001), a type of undirected graphical model, to automatically label fields of contact records. Here, a record is an entire block of a person's contact information, and a field is one element of that record (e.g. COMPANYNAME). We implement several techniques to estimate both field confidence and record confidence, obtaining an average precision of $98 \%$ for fields and $87 \%$ for records.

\section{Conditional Random Fields}

Conditional random fields (Lafferty et al., 2001) are undirected graphical models to calculate the conditional probability of values on designated output nodes given values on designated input nodes. In the special case in which the designated output nodes are linked by edges in a linear chain, CRFs make a first-order Markov independence assumption among output nodes, and thus corre- 
spond to finite state machines (FSMs). In this case CRFs can be roughly understood as conditionally-trained hidden Markov models, with additional flexibility to effectively take advantage of complex overlapping features.

Let $\mathbf{o}=\left\langle o_{1}, o_{2}, \ldots o_{T}\right\rangle$ be some observed input data sequence, such as a sequence of words in a document (the values on $T$ input nodes of the graphical model). Let $\mathcal{S}$ be a set of FSM states, each of which is associated with a label (such as COMPANYNAME). Let $\mathbf{s}=\left\langle s_{1}, s_{2}, \ldots s_{T}\right\rangle$ be some sequence of states (the values on $T$ output nodes). CRFs define the conditional probability of a state sequence given an input sequence as

$$
p_{\Lambda}(\mathbf{s} \mid \mathbf{o})=\frac{1}{Z_{\mathbf{o}}} \exp \left(\sum_{t=1}^{T} \sum_{k} \lambda_{k} f_{k}\left(s_{t-1}, s_{t}, \mathbf{o}, t\right)\right),
$$

where $Z_{\mathbf{o}}$ is a normalization factor over all state sequences, $f_{k}\left(s_{t-1}, s_{t}, \mathbf{o}, t\right)$ is an arbitrary feature function over its arguments, and $\lambda_{k}$ is a learned weight for each feature function. $Z_{\mathrm{o}}$ is efficiently calculated using dynamic programming. Inference (very much like the Viterbi algorithm in this case) is also a matter of dynamic programming. Maximum aposteriori training of these models is efficiently performed by hill-climbing methods such as conjugate gradient, or its improved second-order cousin, limited-memory BFGS.

\section{Field Confidence Estimation}

The Viterbi algorithm finds the most likely state sequence matching the observed word sequence. The word that Viterbi matches with a particular FSM state is extracted as belonging to the corresponding database field. We can obtain a numeric score for an entire sequence, and then turn this into a probability for the entire sequence by normalizing. However, to estimate the confidence of an individual field, we desire the probability of a subsequence, marginalizing out the state selection for all other parts of the sequence. A specialization of Forward-Backward, termed Constrained Forward-Backward (CFB), returns exactly this probability.

Because CRFs are conditional models, Viterbi finds the most likely state sequence given an observation sequence, defined as $s^{*}=\operatorname{argmax}_{\mathbf{s}} p_{\Lambda}(\mathbf{s} \mid \mathbf{o})$. To avoid an exponential-time search over all possible settings of $\mathbf{s}$, Viterbi stores the probability of the most likely path at time $t$ that accounts for the first $t$ observations and ends in state $s_{i}$. Following traditional notation, we define this probability to be $\delta_{t}\left(s_{i}\right)$, where $\delta_{0}\left(s_{i}\right)$ is the probability of starting in each state $s_{i}$, and the recursive formula is:

$$
\delta_{t+1}\left(s_{i}\right)=\max _{s^{\prime}}\left[\delta_{t}\left(s^{\prime}\right) \exp \left(\sum_{k} \lambda_{k} f_{k}\left(s^{\prime}, s_{i}, \mathbf{o}, t\right)\right)\right]
$$

terminating in $s^{*}=\underset{s_{1} \leq s_{i} \leq s_{N}}{\operatorname{argmax}}\left[\delta_{T}\left(s_{i}\right)\right]$.
The Forward-Backward algorithm can be viewed as a generalization of the Viterbi algorithm: instead of choosing the optimal state sequence, Forward-Backward evaluates all possible state sequences given the observation sequence. The "forward values" $\alpha_{t+1}\left(s_{i}\right)$ are recursively defined similarly as in Eq. 2, except the max is replaced by a summation. Thus we have

$$
\alpha_{t+1}\left(s_{i}\right)=\sum_{s^{\prime}}\left[\alpha_{t}\left(s^{\prime}\right) \exp \left(\sum_{k} \lambda_{k} f_{k}\left(s^{\prime}, s_{i}, \mathbf{o}, t\right)\right)\right] .
$$

terminating in $Z_{\mathbf{o}}=\sum_{i} \alpha_{T}\left(s_{i}\right)$ from Eq. 1.

To estimate the probability that a field is extracted correctly, we constrain the Forward-Backward algorithm such that each path conforms to some subpath of constraints $C=\left\langle s_{q} \ldots s_{r}\right\rangle$ from time step $q$ to $r$. Here, $s_{q} \in C$ can be either a positive constraint (the sequence must pass through $s_{q}$ ) or a negative constraint (the sequence must not pass through $s_{q}$ ).

In the context of information extraction, $C$ corresponds to an extracted field. The positive constraints specify the observation tokens labeled inside the field, and the negative constraints specify the field boundary. For example, if we use states names B-TITLE and I-JoBTITLE to label tokens that begin and continue a JOBTITLE field, and the system labels observation sequence $\left\langle o_{2}, \ldots, o_{5}\right\rangle$ as a JobTitle field, then $C=\left\langle s_{2}=\mathrm{B}\right.$-JobTitle, $s_{3}=\ldots=s_{5}=$ I-JobTitle, $s_{6} \neq$ I-JobTitle $\rangle$.

The calculations of the forward values can be made to conform to $C$ by the recursion $\alpha_{q}^{\prime}\left(s_{i}\right)=$

$$
\begin{cases}\sum_{s^{\prime}}\left[\alpha_{q-1}^{\prime}\left(s^{\prime}\right) \exp \left(\sum_{k} \lambda_{k} f_{k}\left(s^{\prime}, s_{i}, \mathbf{o}, t\right)\right)\right] & \text { if } s_{i} \simeq s_{q} \\ 0 & \text { otherwise }\end{cases}
$$

for all $s_{q} \in C$, where the operator $s_{i} \simeq s_{q}$ means $s_{i}$ conforms to constraint $s_{q}$. For time steps not constrained by $C$, Eq. 3 is used instead.

If $\alpha_{t+1}^{\prime}\left(s_{i}\right)$ is the constrained forward value, then $Z_{\mathbf{o}}^{\prime}=\sum_{i} \alpha_{T}^{\prime}\left(s_{i}\right)$ is the value of the constrained lattice, the set of all paths that conform to $C$. Our confidence estimate is obtained by normalizing $Z_{\mathbf{o}}^{\prime}$ using $Z_{\mathbf{o}}$, i.e. $Z_{\mathbf{o}}^{\prime}-Z_{\mathbf{o}}$.

We also implement an alternative method that uses the state probability distributions for each state in the extracted field. Let $\gamma_{t}\left(s_{i}\right)=p\left(s_{i} \mid o_{1}, \ldots, o_{T}\right)$ be the probability of being in state $i$ at time $t$ given the observation sequence. We define the confidence measure GAMMA to be $\prod_{i=u}^{v} \gamma_{i}\left(s_{i}\right)$, where $u$ and $v$ are the start and end indices of the extracted field.

\section{Record Confidence Estimation}

We can similarly use CFB to estimate the probability that an entire record is labeled correctly. The procedure is the same as in the previous section, except that $C$ now specifies the labels for all fields in the record. 
We also implement three alternative record confidence estimates. FIELDPRODUCT calculates the confidence of each field in the record using CFB, then multiplies these values together to obtain the record confidence. FIELDMIN instead uses the minimum field confidence as the record confidence. VITERBIRATIO uses the ratio of the probabilities of the top two Viterbi paths, capturing how much more likely $s^{*}$ is than its closest alternative.

\section{Reranking with Maximum Entropy}

We also trained two conditional maximum entropy classifiers to classify fields and records as being labeled correctly or incorrectly. The resulting posterior probability of the "correct" label is used as the confidence measure. The approach is inspired by results from (Collins, 2000), which show discriminative classifiers can improve the ranking of parses produced by a generative parser.

After initial experimentation, the most informative inputs for the field confidence classifier were field length, the predicted label of the field, whether or not this field has been extracted elsewhere in this record, and the CFB confidence estimate for this field. For the record confidence classifier, we incorporated the following features: record length, whether or not two fields were tagged with the same label, and the CFB confidence estimate.

\section{Experiments}

2187 contact records $(27,560$ words $)$ were collected from Web pages and email and 25 classes of data fields were hand-labeled. ${ }^{1}$ The features for the CRF consist of the token text, capitalization features, 24 regular expressions over the token text (e.g. CONTAInsHyphen), and offsets of these features within a window of size 5 . We also use 19 lexicons, including "US Last Names," "US First Names," and "State Names." Feature induction is not used in these experiments. The CRF is trained on $60 \%$ of the data, and the remaining $40 \%$ is split evenly into development and testing sets. The development set is used to train the maximum entropy classifiers, and the testing set is used to measure the accuracy of the confidence estimates. The CRF achieves an overall token accuracy of 87.32 on the testing data, with a field-level performance of $\mathrm{F} 1=84.11$, precision $=85.43$, and recall $=82.83$.

To evaluate confidence estimation, we use three methods. The first is Pearson's $r$, a correlation coefficient ranging from -1 to 1 that measures the correlation between a confidence score and whether or not the field (or record) is correctly labeled. The second is average precision, used in the Information Retrieval community

\footnotetext{
${ }^{1}$ The 25 fields are: FirstName, MiddleName, LastName, NickName, Suffix, Title, JobTitle, CompanyName, Department, AddressLine, City1, City2, State, Country, PostalCode, HomePhone, Fax, CompanyPhone, DirectCompanyPhone, Mobile, Pager, VoiceMail, URL, Email, InstantMessage
}

\begin{tabular}{|c|c|c|}
\hline & Pearson's r & Avg. Prec \\
\hline CFB & $\mathbf{. 5 7 3}$ & $\mathbf{. 9 7 6}$ \\
MaxEnt & $\mathbf{. 5 7 1}$ & $\mathbf{. 9 7 6}$ \\
Gamma & .418 & .912 \\
Random & .012 & .858 \\
WorstCase & - & .672 \\
\hline
\end{tabular}

Table 1: Evaluation of confidence estimates for field confidence. CFB and MAXENT outperform competing methods.

\begin{tabular}{|c|c|c|}
\hline & Pearson's r & Avg. Prec \\
\hline CFB & $\mathbf{. 6 2 6}$ & $\mathbf{. 8 6 3}$ \\
MaxEnt & $\mathbf{. 6 3 0}$ & $\mathbf{. 8 6 7}$ \\
FieldProduct & .608 & .858 \\
FieldMin & .588 & .843 \\
ViterbiRatio & .313 & .842 \\
Random & .043 & .526 \\
WorstCase & - & .304 \\
\hline
\end{tabular}

Table 2: Evaluation of confidence estimates for record confidence. CFB, MAXENT again perform best.

to evaluate ranked lists. It calculates the precision at each point in the ranked list where a relevant document is found and then averages these values. Instead of ranking documents by their relevance score, here we rank fields (and records) by their confidence score, where a correctly labeled field is analogous to a relevant document. WORSTCASE is the average precision obtained by ranking all incorrect instances above all correct instances. Tables 1 and 2 show that CFB and MAXENT are statistically similar, and that both outperform competing methods. Note that WORSTCASE achieves a high average precision simply because so many fields are correctly labeled. In all experiments, RANDOM assigns confidence values chosen uniformly at random between 0 and 1 .

The third measure is an accuracy-coverage graph. Better confidence estimates push the curve to the upper-right. Figure 1 shows that CFB and MAXENT dramatically outperform GAMMA. Although omitted for space, similar results are also achieved on a noun-phrase chunking task (CFB $r=.516$, GAMMA $r=.432$ ) and a named-entity extraction task (CFB $r=.508$, GAMMA $r=.480$ ).

\section{Related Work}

While there has been previous work using probabilistic estimates for token confidence, and heuristic estimates for field confidence, to the best of our knowledge this paper is the first to use a sound, probabilistic estimate for confidence of multi-word fields and records in information extraction.

Much of the work in confidence estimation for IE has been in the active learning literature. Scheffer et al. (2001) derive confidence estimates using hidden Markov models in an information extraction system. However, they do not estimate the confidence of entire fields, only singleton tokens. They estimate 


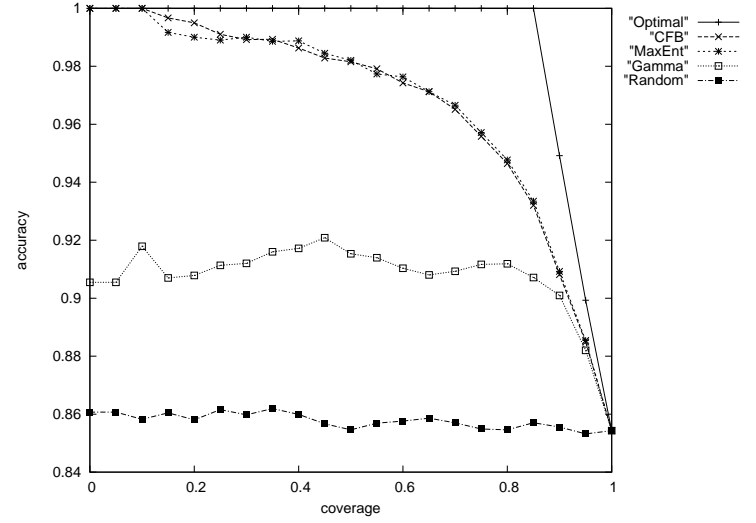

Figure 1: The precision-recall curve for fields shows that CFB and MAXENT outperform GAMMA.

the confidence of a token by the difference between the probabilities of its first and second most likely labels, whereas CFB considers the full distribution of all suboptimal paths. Scheffer et al. (2001) also explore an idea similar to CFB to perform Baum-Welch training with partially labeled data, where the provided labels are constraints. However, these constraints are again for singleton tokens only.

Rule-based extraction methods (Thompson et al., 1999) estimate confidence based on a rule's coverage in the training data. Other areas where confidence estimation is used include document classification (Bennett et al., 2002), where classifiers are built using meta-features of the document; speech recognition (Gunawardana et al., 1998), where the confidence of a recognized word is estimated by considering a list of commonly confused words; and machine translation (Gandrabur and Foster, 2003), where neural networks are used to learn the probability of a correct word translation using text features and knowledge of alternate translations.

\section{Conclusion}

We have shown that CFB is a mathematically and empirically sound confidence estimator for finite state information extraction systems, providing strong correlation with correctness and obtaining an average precision of $97.6 \%$ for estimating field correctness. Unlike methods margin maximization methods such as SVMs and $\mathrm{M}^{3} \mathrm{Ns}$ (Taskar et al., 2003), CRFs are trained to maximize conditional probability and are thus more naturally appropriate for confidence estimation. Interestingly, reranking by MAXENT does not seem to improve performance, despite the benefit Collins (2000) has shown discriminative reranking to provide generative parsers. We hypothesize this is because CRFs are already discriminative (not joint, generative) models; furthermore, this may suggest that future discriminative parsing methods will also have the benefits of discriminative reranking built-in directly.

\section{Acknowledgments}

We thank the reviewers for helpful suggestions and references. This work was supported in part by the Center for Intelligent Information Retrieval, by the Advanced Research and Development Activity under contract number MDA90401-C-0984, by The Central Intelligence Agency, the National Security Agency and National Science Foundation under NSF grant \#IIS-0326249, and by the Defense Advanced Research Projects Agency, through the Department of the Interior, NBC, Acquisition Services Division, under contract number NBCHD030010.

\section{References}

Paul N. Bennett, Susan T. Dumais, and Eric Horvitz. 2002. Probabilistic combination of text classifiers using reliability indicators: models and results. In Proceedings of the 25th annual international ACM SIGIR conference on Research and development in information retrieval, pages 207-214. ACM Press.

Michael Collins. 2000. Discriminative reranking for natural language parsing. In Proc. 17th International Conf. on Machine Learning, pages 175-182. Morgan Kaufmann, San Francisco, CA.

Simona Gandrabur and George Foster. 2003. Confidence estimation for text prediction. In Proceedings of the Conference on Natural Language Learning (CoNLL 2003), Edmonton, Canada.

A. Gunawardana, H. Hon, and L. Jiang. 1998. Word-based acoustic confidence measures for large-vocabulary speech recognition. In Proc. ICSLP-98, pages 791-794, Sydney, Australia.

Trausti Kristjannson, Aron Culotta, Paul Viola, and Andrew McCallum. 2004. Interactive information extraction with conditional random fields. To appear in Nineteenth National Conference on Artificial Intelligence (AAAI 2004).

John Lafferty, Andrew McCallum, and Fernando Pereira. 2001. Conditional random fields: Probabilistic models for segmenting and labeling sequence data. In Proc. 18th International Conf. on Machine Learning, pages 282-289. Morgan Kaufmann, San Francisco, CA.

Andrew McCallum and David Jensen. 2003. A note on the unification of information extraction and data mining using conditional-probability, relational models. In IJCAIO3 Workshop on Learning Statistical Models from Relational Data.

Tobias Scheffer, Christian Decomain, and Stefan Wrobel. 2001. Active hidden markov models for information extraction. In Advances in Intelligent Data Analysis, 4th International Conference, IDA 2001.

Ben Taskar, Carlos Guestrin, and Daphne Koller. 2003. Maxmargin markov networks. In Proceedings of Neural Information Processing Systems Conference.

Cynthia A. Thompson, Mary Elaine Califf, and Raymond J. Mooney. 1999. Active learning for natural language parsing and information extraction. In Proc. 16th International Conf. on Machine Learning, pages 406-414. Morgan Kaufmann, San Francisco, CA. 